Running head: DISCRIMINATING FACIAL BEAUTY IN PERIPHERY

\title{
I Know You Are Beautiful Even Without Looking at You: Discrimination of Facial Beauty in Peripheral Vision
}

\author{
Kun Guo ${ }^{1}$, Chang Hong Liu ${ }^{2}$, and Hettie Roebuck ${ }^{1}$ \\ ${ }^{1}$ University of Lincoln, UK; and ${ }^{2}$ University of Hull, UK
}

Correspondence

Chang Hong Liu, PhD

Department of Psychology

University of Hull, Hull HU6 7RX, United Kingdom

Tel: +44-1482-465572

E-mail: c.h.liu@hull.ac.uk 


\begin{abstract}
Prior research suggests that facial attractiveness may capture attention at parafovea. However, little is known about how well facial beauty can be detected at parafoveal and peripheral vision. Participants in this study judged relative attractiveness of a face pair presented simultaneously at several eccentricities from the central fixation. The results show that beauty is not only detectable at parafovea but also at periphery. The discrimination performance at parafovea was indistinguishable from the performance around the fovea. Moreover, performance was well above chance even at the periphery. The results show that the visual system is able to use the low spatial frequency information to appraise attractiveness. These findings not only provide an explanation for why a beautiful face could capture attention when central vision is already engaged elsewhere, but also reveal the potential means by which a crowd of faces is quickly scanned for attractiveness.
\end{abstract}

Keywords: Facial attractiveness; Fovea; Parafovea; Peripheral vision 
Beauty is difficult to ignore even when attention is already engaged in a different task. Sui and Liu (2009) recently demonstrated that a task-irrelevant attractive face presented at parafovea can compete with an ongoing task for spatial attention, suggesting coarse-scale facial information outside the central vision is sufficient for automatic appraisal of facial attractiveness (also see Bachmann 2007, for a study of coarse-scale information for facial beauty discrimination at central vision). However, because the face in the Sui and Liu study was presented at a fixed distance $\left(3.6^{\circ}\right)$ from the fixation, it was not clear whether this effect also extends to far parafoveal and even peripheral vision. Neither was it clear whether the accuracy of attractiveness discrimination at parafovea is as reliable as in the fovea. Here we examined these questions systematically. Participants judged relative attractiveness of a face pair presented simultaneously at several eccentricities from the central fixation.

It is well known that when a pair of faces is presented, the more attractive face of the two tends to draw greater attention and more inspections (Leder et al 2010; Shimojo et al 2003). In these and other similar studies, researchers have focused on how central vision is engaged in the processing of facial beauty. Their results demonstrate that attractive faces tend to dictate preferential eye gaze and fixations, which result in foveation of the preferred face for more detailed information processing.

Unlike most prior studies, the main interest of the present paper is the discrimination of facial beauty beyond the central vision. Given that a vast portion of our visual environment falls outside the foveal region, being able to monitor and assess the visual information and events in the parafoveal and peripheral regions is critical for adaptive reasons. The same ability is also necessary for covert attention where the focus 
of attention often does not coincide with the high-resolution fovea. Prior research has revealed that the ability to discriminate a face identity drops rapidly as a function of eccentricity (Mäkelä et al 2001; Melmoth et al 2000). Moreover, past studies have identified a band of middle frequencies (approximately 8-16 cycles per face) that is critical for face recognition (Bachmann 1991; Costen et al 1994, 1996; Fiorentini et al 1983; Harmon 1971; Harmon and Julesz 1973; Peli et al 1994). However, discrimination or appraisal of facial beauty may require less spatial details than the discrimination of facial identities. There is evidence that low spatial frequency information is often sufficient for discriminating facial beauty or some common facial expressions such as anger and fear presented at the central vision (Bachmann 2007; Schyns and Oliva 1999; Vuilleumier et al 2003). Perhaps the early stage of attractiveness appraisal can rely on low spatial frequency information that is available at the periphery. To our knowledge, the present study was the first attempt to test this hypothesis at the periphery.

To prevent saccadic eye movements to either face image, we presented each face pair on the two sides of the central fixation for a brief $100 \mathrm{~ms}$. The participant's fixation was monitored by an eye tracker during the task. Apart from pairing an attractive face with an unattractive one based on pre-rated scores, we also asked our participants to provide their own attractiveness rating after they had completed the discrimination task. We predicted a stronger effect of eccentricity on discrimination of facial beauty when the difference in attractiveness between two faces is deemed small by participants. 


\section{Method}

Materials

The face database was obtained from University of St. Andrews. All faces were frontal-view Caucasians with neutral expression, and were pre-rated for attractiveness on a 7-point scale. We chose 17 attractive (mean rating $=4.55, S D=0.30$ ) and 17 unattractive (mean rating $=2.03, S D=0.22$ ) faces for this study. The chosen faces had no visible facial marks or distinctive hair style (e.g. fringe). The greyscale faces were then cropped to remove external features and to fit within an oval window subtending $8.0 \times$ $6.2^{\circ}$ of visual angle, equivalent to the size of a real face shown at a distance during typical conversation (Henderson et al 2005). All images were scaled to a same mean luminance and root-mean-square contrast. Each attractive face was randomly paired with an unattractive face. These two faces were then presented bilaterally at equidistance from a central fixation. The three levels of eccentricity (the distance between the inner edge of a face image and the fixation) were $2^{\circ}, 5^{\circ}$ and $10^{\circ}$, which were chosen to probe foveal, parafoveal and peripheral vision, respectively. Each face was used three or four times in different attractive/unattractive combinations to create 60 pairs (20 pairs per eccentricity). Each pair was presented twice but in a separate block to counterbalance left/right presentation location. This amounted to a total of 120 trials.

\section{Participants}

Seventeen Caucasian participants $(12$ women, mean age $=25, S D=10)$ with normal vision viewed the stimulus display binocularly from $57 \mathrm{~cm}$ on a chin rest. Informed consent was obtained from each participant, and all procedures complied with 
the World Medical Association Helsinki Declaration as revised in October 2008. During the experiment, their fixations were monitored using a Video Eyetracker Toolbox with 50 $\mathrm{Hz}$ sampling frequency and $0.25^{\circ}$ accuracy (Cambridge Research Systems). The stimulus was presented through a ViSaGe graphics system on a gamma-corrected monitor (1024× 768 pixels, $100 \mathrm{~Hz}$ frame rate, Mitsubishi) with grey background.

\section{Procedure}

Each trail started with a warning tune, followed by a 1-sec central fixation. A pair of faces was then presented for $100 \mathrm{~ms}$. Participants were instructed to maintain their fixation and to respond as accurately and as quickly as possible by pressing one of the two buttons to indicate whether the left or right face was more attractive. No feedback was given. The inter-trial interval was $1.5 \mathrm{sec}$.

To examine how the participant's own judgement of attractiveness affects the discrimination performance, all participants also performed a separate, self-paced task where the same face pairs were presented side by side with $1^{\circ}$ gap in a randomised order. The task was to indicate which face within the pair was more attractive and how different the two faces was in attractiveness on a 3 -point scale $(1=$ slightly different, $3=$ very different). The task was always performed after the discrimination task. For these manipulated face images, all participants' judgements in the rating task were consistent with the answers defined by the pre-rating scores, $t(16)=1.03, p=.32$. That is, within a face pair, the face with higher pre-rating score in attractiveness was also judged to be more attractive by our participants (mean attractiveness discrimination performance $=$ $99.9 \%, S D=0.40)$. 


\section{Results}

In total $2.8 \%$ of the trials were excluded from analysis because the eye drifted $1^{\circ}$ or more away from the central fixation during the face presentation. Results in Figure 1A show that the attractiveness discrimination rate was significantly affected by the eccentricity, $F(2,48)=16.8, p<.001, \eta_{\mathrm{p}}{ }^{2}=0.41$. The performance was indistinguishable for faces presented at $2^{\circ}$ and $5^{\circ}$ eccentricity $(>80 \%), t(16)=1.4, p=.18$. Performance at $10^{\circ}$ eccentricity ( $>65 \%$ ) was significantly lower than these but was clearly above-chance, $t(16)=7.13, p<.001$. Eccentricity had no significant impact on the reaction time data, $F(2,48)=1.33, p=.28, \eta_{\mathrm{p}}^{2}=0.05$.

Discrimination performance was also analyzed according to the perceived difference between the attractiveness of two simultaneously presented faces (Figure 1B). There was again a significant main effect of eccentricity, $F(2,133)=19.45, p<.001, \eta_{\mathrm{p}}{ }^{2}$ $=0.23$. The main effect of perceived attractiveness difference approached the level of significance, $F(2,133)=2.35 p=.10, \eta_{\mathrm{p}}^{2}=0.03$, suggesting an easier discrimination when one face in a pair was clearly more attractive than another. The interaction between the two variables was not significant, $F(4,133)=0.59, p=.67, \eta_{\mathrm{p}}{ }^{2}=0.02$. Again, analysis of the reaction time data did not reveal any significant result.

\section{Discussion}

Prior research suggests that facial attractiveness may be detectable at parafovea (Sui and Liu 2009). Our new results show that beauty is not only detectable at parafovea but also at periphery. Furthermore, the attractiveness discrimination at parafovea $\left(5^{\circ}\right)$ was indistinguishable from the performance around the fovea $\left(2^{\circ}\right)$. Comparing to parafovea, 
the performance at periphery $\left(10^{\circ}\right)$ was clearly worsened. However, it is remarkable that even here the discrimination performance was still well above-chance. This suggests that our visual system is able to use the low spatial frequency information to perform rapid appraisal of faces for attractiveness. This is consistent with prior observation that physical carriers of facial beauty are originally present in the coarse configural properties of faces (Bachmann 2007). Finally, our results show that such a judgment of relative attractiveness at the periphery is sensitive to how much a face is more attractive than the other. Discrimination errors appeared to be higher when the perceived difference in attractiveness between the two faces was smaller. This finding suggests that finer discrimination or ranking of facial beauty may require more facial details contained in higher spatial frequencies. The capacity of the peripheral vision may be limited to categorical attractive judgements.

These findings not only provide an explanation for why a beautiful face could capture attention when central vision is already engaged elsewhere, but also reveal the potential means by which a crowd of faces is quickly scanned and appraised for attractiveness. It is interesting to note that the participants had little difficulty to appraise two briefly flashed faces very quickly. This means the judgement of relative attractiveness may not require foveation when a face is clearly more attractive than the other. Moreover, it means that a quick sampling of attractive faces from a crowd is possible because a relatively small number of fixations would be sufficient if it is unnecessary to foveate on each individual faces.

The biological significance of beauty detection may be comparable to some wellstudied facial cues such as threatening and fearful expressions that are also known to be 
detected rapidly at extrafovea (Goren and Wilson 2006; Leppanen and Nelson 2009). Apart from potential danger, our study shows that peripheral vision is also sensitive to potential reward. The visual system may be evolved to rapidly detect attractiveness in the periphery, which may trigger the act of foveation for detailed visual analysis. In other words, an extrafoveal detection of facial beauty may be a precursor for eye gaze, preferential looking, and other deeper visual and attentional processing at the fovea. 


\section{References}

Bachmann T, 1991 "Identification of spatially quantised tachistoscopic images of faces: How many pixels does it take to carry identity? " European Journal of Cognitive Psychology 3 85-103

Bachmann T, 2007 "When beauty breaks down: Investigation of the effect of spatial quantisation on aesthetic evaluation of facial images" Perception 36 840-849

Costen N P, Parker D M, Craw I, 1994 "Spatial content and spatial quantisation effects in face recognition” Perception 23 129-146

Costen N P, Parker D M, Craw I, 1996 "Effects of high-pass and low-pass spatial filtering on face identification” Perception \& Psychophysics 58 602-612

Fiorentini A, Maffei L, Sandim G, 1983 "The role of high spatial frequencies in face perception" Perception 12 195-201

Goren D, Wilson H R, 2006 “Quantifying facial expression recognition across viewing conditions" Vision Research 46 1253-1262

Harmon L D, 1971 “The recognition of faces” Scientific American 229 70-82

Harmon L D, Julesz B, 1973 "Masking in visual recognition: Effects of two-dimensional filtered noise" Science 180 1194-1197

Henderson J M, Williams C C, Falk R J, 2005 “Eye movements are functional during face learning” Memory \& Cognition 33 98-106

Leder H, Tinio P P L, Fuchs I M, Bohrn I, 2010 "When attractiveness demands longer looks: The effects of situation and gender" The Quarterly Journal of Experimental Psychology 63 1858-1871 
Leppanen J M, Nelson C A, 2009 "Tuning the developing brain to social signals of emotions" Nature Reviews Neuroscience 10 37-47

Mäkelä P, Näsänen R, Rovamo J, Melmoth D, 2001 "Identification of facial images in peripheral vision” Vision Research 41 599-610

Melmoth D R, Kukkonen H T, Mäkelä P K, Rovamo J M, 2000 “The effect of contrast and size scaling on face perception in foveal and extrafoveal vision" Investigative Ophthalmology \& Visual Science 41 2811-2819

Peli E, Lee E, Trempe C L, Buzney S, 1994 "Image enhancement for the visually impaired: The effects of enhancement on face recognition" Journal of the Optical Society of America A-Optics \& Image Science 11 1929-1939

Schyns P G, Oliva A, 1999 "Dr. Angry and Mr. Smile: When categorization flexibly modifies the perception of faces in rapid visual presentations" Cognition 69243 265

Shimojo S, Simion C, Shimojo E, Scheier C, 2003 "Gaze bias both reflects and influences preference" Nature Neuroscience 6 1317-1322

Sui J, Liu C H, 2009 "Can beauty be ignored? Effects of facial attractiveness on covert attention” Psychonomic Bulletin \& Review 16 276-281

Vuilleumier P, Armony J L, Driver J, Dolan R J, 2003 "Distinct spatial frequency sensitivities for processing faces and emotional expressions" Nature Neuroscience $6624-631$ 


\section{Figure 1}
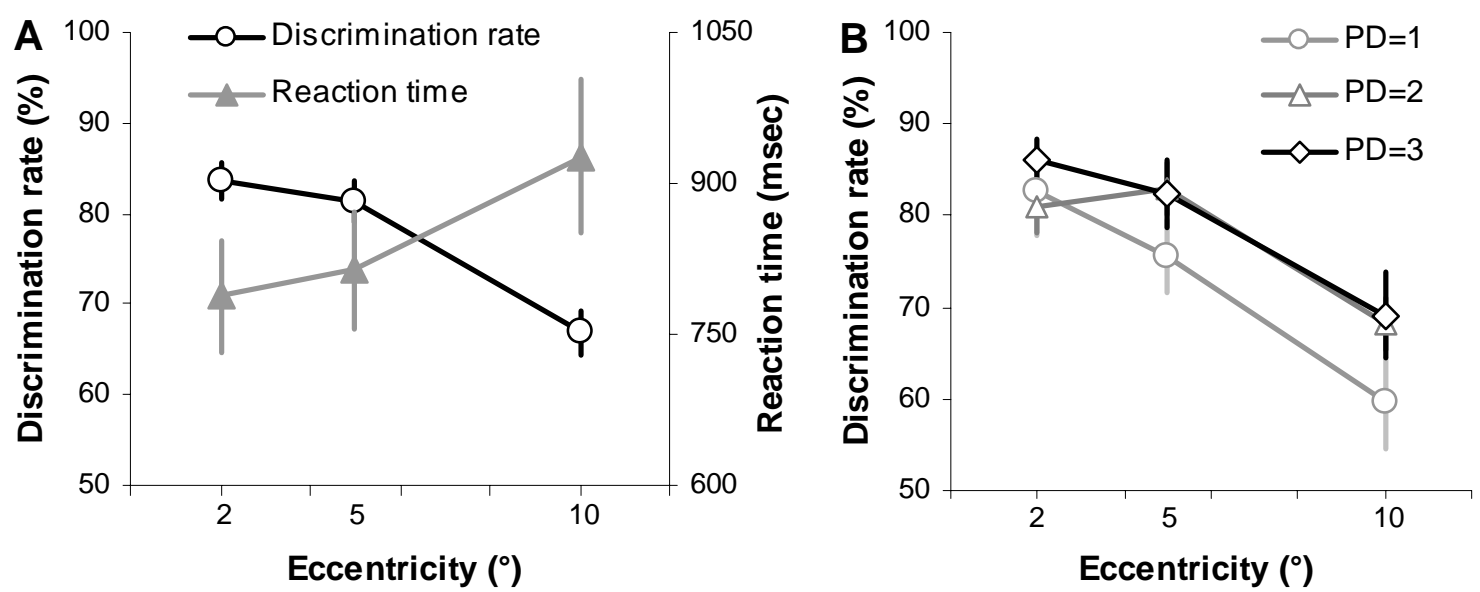

Figure 1. A. Discrimination rate and reaction time for attractive faces as a function of eccentricity. B. Influence of perceived difference (PD) in attractiveness on discrimination rate for attractive faces presented at $2^{\circ}, 5^{\circ}$ and $10^{\circ}$ eccentricities. Error bars show standard errors. 